\title{
The Dynamics of a Discrete Fractional-Order Logistic Growth Model with Infectious Disease
}

\author{
Hasan S. Panigoro ${ }^{1, *}$ \& Emli Rahmi ${ }^{2}$ \\ ${ }^{1,2}$ Department of Mathematics, State University of Gorontalo, Bone Bolango 96119, Indonesia \\ *Corresponding author. Email: hspanigoro@ung.ac.id
}

\begin{abstract}
In this paper, we study the dynamics of a discrete fractional-order logistic growth model with infectious disease. We obtain the discrete model by applying the piecewise constant arguments to the fractional-order model. This model contains three fixed points namely the origin point, the disease-free point, and the endemic point. We confirm that the origin point is always exists and unstable, the disease-free point is always exists and conditionally stable, and the endemic point is conditionally exists and stable. We also investigate the existence of forward, period-doubling, and Neimark-Sacker bifurcation. The numerical simulations are also presented to confirm the analytical results. We also show numerically the existence of period-3 solution which leads to the occurrence of chaotic behavior.
\end{abstract}

Keywords: logistic growth model; infectious disease; piecewise constant arguments; bifurcation.

\section{$1 \quad$ Introduction}

The infectious disease is the best opponents of the human's existence [1]. Therefore, many scientists focus in studying the optimal solutions to protect human from the infectious disease both theoretically and practically. In mathematical point of view, the infectious disease is investigated by using mathematical modeling. One of well-liked mathematical approach is the deterministic modeling using differential equation. Especially for the mathematical modeling in epidemiological problem, the model proposed by Kermack-MacKendrick [2] becomes the most famous among. This model is referenced by many scholars in developing new models which associated with the specific phenomena. For example, see [2]-[8] and references cited therein.

In the past few years, many scholars are interested to study the epidemiological model with the fractional-order derivative rather than the integer-order derivative. The models with fractional-order derivative are considered able to present more realistic epidemiological phenomena due to its capability in integrating the memory effects as well as the history of the biological properties ([9]-[11]). Although the mathematical modeling with fractional-order derivative is growing rapidly, there are several studies investigate the biological circumstance in the discrete model, see [12]-[14] and references therein. This modeling is considered plausible since the actual conditions are provided in the 
discrete data. Moreover, the discrete model also attractive for some researchers inasmuch as it gives rich dynamics rather than the continuous model. The far-famed discretization scheme to obtain the discrete model from the fractional-order derivative model is the piecewise constant arguments (PWCA). The PWCA scheme can be found in [15] and [16].

Table 1 Variables and parameters description

\begin{tabular}{cl}
\hline Variables and parameters & Description \\
\hline$S$ & Susceptible population \\
$I$ & Infected population \\
$r$ & Intrinsic growth rate \\
$K$ & Environmental carrying capacity \\
$\beta$ & Infection rate \\
$\omega$ & Recovery rate \\
$\delta$ & Death rate \\
\hline
\end{tabular}

In this paper, we investigate the dynamics of a discrete model constructed by PWCA from the fractional-order model proposed by Panigoro \& Rahmi [6]. As far as we know, the discrete form of this model has never been studied and investigated. We interest to study the discrete form of this model by taking into account the proposed model has a simple way in describing the infectious disease using logistic equation. The fairly similar model is given by Abdelaziz et al. [12]. The different lies on the growth rate of susceptible population and the ability to recover of infected population. Model in [12] using constant growth rate and the infected population cannot recover from the disease while model in [6] using logistic growth rate and the infect population has the ability to recover.

This paper is organized as follows. In Section 2, the model formulation including the fractional model and its discretization process with PWCA is given. Furthermore, we discuss the existence of fixed point and the local stability in Section 3. In Section 4, the existence of several bifurcations such as forward, period-doubling, and Neimark-Sacker bifurcations are discussed. We give the numerical simulations including the influence of the step-size and the infection rate in Section 5. In this Section, we also give short discussion about the existence of chaotic behavior near period-3 solution. In Section 6, we end our works by giving conclusion.

\section{Model Formulation}

The fractional-order logistic growth model with infectious disease proposed by Panigoro $\&$ Rahmi [6] is given by the following equations. 


$$
\begin{aligned}
{ }^{c} D_{t}^{\alpha} S & =r S\left(1-\frac{S+I}{K}\right)-\beta S I+\omega I, \\
{ }^{c} D_{t}^{\alpha} I & =\beta S I-(\omega+\delta) I,
\end{aligned}
$$

where the definition of Caputo fractional-order derivative ${ }^{C} D_{t}^{\alpha}$ is given by

$$
{ }^{C} D_{t}^{\alpha} u(t)=\mathfrak{J}^{1-\alpha} u^{\prime}(t), 0<\alpha \leq 1 .
$$

$I^{\theta}$ denotes integral operator of Riemann-Liouville with order $\theta$ defined by

$$
\mathcal{J}^{\theta} v(t)=\frac{1}{\Gamma(\theta)} \int_{0}^{t}(t-s)^{\theta-1} v(s) d s
$$

with $t \geq 0, u \in C^{n}([0,+\infty), \mathbb{R})$, and $\Gamma(\cdot)$ is the Euler's Gamma function [17],[18]. The description of variables and parameters are given in Table 1. To obtain the discrete model, we apply the piecewise constant arguments (PWCA) adapted from [19]-[22]. The PWCA of model (1) is given by the following equations.

$$
\begin{aligned}
{ }^{C} D_{t}^{\alpha} S= & r S\left(\left[\frac{t}{h}\right] h\right)\left(1-\frac{S\left(\left[\frac{t}{h}\right] h\right)+I\left(\left[\frac{t}{h}\right] h\right)}{K}\right) \\
& -\beta S\left(\left[\frac{t}{h}\right] h\right) I\left(\left[\frac{t}{h}\right] h\right)+\omega I\left(\left[\frac{t}{h}\right] h\right), \\
{ }^{C} D_{t}^{\alpha} I= & \beta S\left(\left[\frac{t}{h}\right] h\right) I\left(\left[\frac{t}{h}\right] h\right)-(\omega+\delta) I\left(\left[\frac{t}{h}\right] h\right),
\end{aligned}
$$

with initial condition $x(0)=x_{0}$ and $y(0)=y_{0}$. Now, for $t \in[0, h), \frac{t}{h} \in[0,1)$, we have

$$
\begin{aligned}
{ }^{C} D_{t}^{\alpha} S & =r S_{0}\left(1-\frac{S_{0}+I_{0}}{K}\right)-\beta S_{0} I_{0}+\omega I_{0}, \\
{ }^{C} D_{t}^{\alpha} I & =\beta S_{0} I_{0}-(\omega+\delta) I_{0},
\end{aligned}
$$

which gives solutions

$$
\begin{aligned}
& S_{1}=S_{0}+\mathcal{J}^{\alpha}\left[r S_{0}\left(1-\frac{S_{0}+I_{0}}{K}\right)-\beta S_{0} I_{0}+\omega I_{0}\right], \\
& I_{1}=I_{0}+\mathcal{J}^{\alpha}\left[\beta S_{0} I_{0}-(\omega+\delta) I_{0}\right] .
\end{aligned}
$$

By employing eq. (3) to eq. (5), we get solutions 


$$
\begin{aligned}
S_{1} & =S_{0}+\frac{t^{\alpha}}{\Gamma(1+\alpha)}\left[r S_{0}\left(1-\frac{S_{0}+I_{0}}{K}\right)-\beta S_{0} I_{0}+\omega I_{0}\right] \\
I_{1} & =I_{0}+\frac{t^{\alpha}}{\Gamma(1+\alpha)}\left[\beta S_{0} I_{0}-(\omega+\delta) I_{0}\right] .
\end{aligned}
$$

Next, for $t \in[h, 2 h), \frac{t}{h} \in[1,2)$, from eqs. (4), we get

$$
\begin{aligned}
{ }^{C} D_{t}^{\alpha} S & =r S_{1}\left(1-\frac{S_{1}+I_{1}}{K}\right)-\beta S_{1} I_{1}+\omega I_{1}, \\
{ }^{c} D_{t}^{\alpha} I & =\beta S_{1} I_{1}-(\omega+\delta) I_{1},
\end{aligned}
$$

where the solutions are

$$
\begin{aligned}
S_{2} & =S_{1}+\mathcal{J}^{\alpha}\left[r S_{1}\left(1-\frac{S_{1}+I_{1}}{K}\right)-\beta S_{1} I_{1}+\omega I_{1}\right], \\
I_{2} & =I_{1}+\mathcal{J}^{\alpha}\left[\beta S_{1} I_{1}-(\omega+\delta) I_{1}\right] .
\end{aligned}
$$

Eqs. (6) have solutions

$$
\begin{aligned}
S_{2} & =S_{1}+\frac{(t-h)^{\alpha}}{\Gamma(1+\alpha)}\left[r S_{1}\left(1-\frac{S_{1}+I_{1}}{K}\right)-\beta S_{1} I_{1}+\omega I_{1}\right] \\
I_{2} & =I_{1}+\frac{(t-h)^{\alpha}}{\Gamma(1+\alpha)}\left[\beta S_{1} I_{1}-(\omega+\delta) I_{1}\right] .
\end{aligned}
$$

Again, for $t \in[2 h, 3 h), \frac{t}{h} \in[2,3)$, according to eqs. (4), we acquire

$$
\begin{aligned}
{ }^{C} D_{t}^{\alpha} S & =r S_{2}\left(1-\frac{S_{2}+I_{2}}{K}\right)-\beta S_{2} I_{2}+\omega I_{2}, \\
{ }^{C} D_{t}^{\alpha} I & =\beta S_{2} I_{2}-(\omega+\delta) I_{2} .
\end{aligned}
$$

Therefore, we achieve

$$
\begin{aligned}
S_{3} & =S_{2}+\mathcal{J}^{\alpha}\left[r S_{2}\left(1-\frac{S_{2}+I_{2}}{K}\right)-\beta S_{2} I_{2}+\omega I_{2}\right], \\
I_{3} & =I_{2}+\mathcal{J}^{\alpha}\left[\beta S_{2} I_{2}-(\omega+\delta) I_{2}\right] .
\end{aligned}
$$

By using eq. (3), we achieve solutions for eqs. (7) as follows.

$$
\begin{aligned}
S_{3} & =S_{1}+\frac{(t-h)^{\alpha}}{\Gamma(1+\alpha)}\left[r S_{1}\left(1-\frac{S_{1}+I_{1}}{K}\right)-\beta S_{1} I_{1}+\omega I_{1}\right] \\
I_{3} & =I_{1}+\frac{(t-h)^{\alpha}}{\Gamma(1+\alpha)}\left[\beta S_{1} I_{1}-(\omega+\delta) I_{1}\right] .
\end{aligned}
$$


Repeating this scheme for $n$ times, whe have $t \in[n h,(n+1) h), \frac{t}{h} \in[n, n+1)$, and hence

$$
\begin{aligned}
S_{n+1} & =S_{n}+\mathcal{J}^{\alpha}\left[r S_{n}\left(1-\frac{S_{n}+I_{n}}{K}\right)-\beta S_{n} I_{n}+\omega I_{n}\right], \\
I_{n+1} & =I_{n}+\mathcal{J}^{\alpha}\left[\beta S_{n} I_{n}-(\omega+\delta) I_{n}\right] .
\end{aligned}
$$

where according to eq. (3), eqs. (8) have solutions

$$
\begin{aligned}
S_{n+1} & =S_{n}+\frac{(t-n h)^{\alpha}}{\Gamma(1+\alpha)}\left[r S_{n}\left(1-\frac{S_{n}+I_{n}}{K}\right)-\beta S_{n} I_{n}+\omega I_{n}\right], \\
I_{n+1} & =I_{n}+\frac{(t-n h)^{\alpha}}{\Gamma(1+\alpha)}\left[\beta S_{n} I_{n}-(\omega+\delta) I_{n}\right] .
\end{aligned}
$$

Finally, Let $t \rightarrow(n+1) h$. Then the eqs. (9) are reduced to a discrete model as follows.

$$
\begin{aligned}
S_{n+1} & =S_{n}+\frac{h^{\alpha}}{\Gamma(1+\alpha)}\left[r S_{n}\left(1-\frac{S_{n}+I_{n}}{K}\right)-\beta S_{n} I_{n}+\omega I_{n}\right], \\
I_{n+1} & =I_{n}+\frac{h^{\alpha}}{\Gamma(1+\alpha)}\left[\beta S_{n} I_{n}-(\omega+\delta) I_{n}\right] .
\end{aligned}
$$

The model (10) is the discrete form of model (1) obtained by PWCA. Denote if $\alpha \rightarrow 1$ then we have the forward Euler discretization. Furthermore, we study the dynamics of model (10) including the existence of fixed points and their local stability.

\section{$3 \quad$ Fixed Points and Their Local Stability}

In this section, the existence of fixed points and their local stability is investigated. To support our analytical study, the following Lemmas are given.

Lemma 1. [12] Let $\lambda_{1}$ and $\lambda_{2}$ are the eigen values of the Jacobian matrix evaluated at a fixed point $x^{*} \in \mathbb{R}^{2}$ of a difference equation $x_{n+1}=f\left(x_{n}\right), x \in \mathbb{R}^{2}$. Then the fixed point $x^{*}$ is

(i) a sink (locally asymptotically stable) if $\left|\lambda_{1}\right|<1$ and $\left|\lambda_{2}\right|<1$; or

(ii) a source (unstable) if $\left|\lambda_{1}\right|>1$ and $\left|\lambda_{2}\right|>1$; or

(iii) a saddle (unstable) if $\left|\lambda_{1}\right|<1$ and $\left|\lambda_{2}\right|>1$, or if $\left|\lambda_{1}\right|>1$ and $\left|\lambda_{2}\right|<1$; or

(iv) a non-hyperbolic if $\left|\lambda_{1}\right|=1$ or $\left|\lambda_{2}\right|=1$.

Lemma 2. [12] Let $F(\lambda)=\lambda^{2}-\operatorname{Tr} \lambda+$ Det. Suppose that (1) $>0, \lambda_{1}, \lambda_{2}$ are the two roots of $F(\lambda)=0$. Then

(i) $\left|\lambda_{1}\right|<1$ and $\left|\lambda_{2}\right|<1$ if and only if $F(-1)>0$ and Det $<1$,

(ii) $\left|\lambda_{1}\right|>1$ and $\left|\lambda_{2}\right|>1$ if and only if $F(-1)>0$ and Det $>1$, 
(iii) $\left|\lambda_{1}\right|<1$ and $\left|\lambda_{2}\right|>1$, or $\left|\lambda_{1}\right|>1$ and $\left|\lambda_{2}\right|<1$ if and only if $F(-1)<0$,

(iv) $\lambda_{1}=-1$ and $\lambda_{2} \neq 1$ if and only if $F(-1)=0$ and $\operatorname{Tr} \neq 0,2$, and

(v) $\lambda_{1}$ and $\lambda_{2}$ are complex and $\left|\lambda_{1}\right|=\left|\lambda_{2}\right|=1$ if and only if $\operatorname{Tr}^{2}-4$ Det $<0$ and Det $=1$.

Furthermore, we first identify the fixed points by solving the following equations.

$$
\begin{aligned}
S & =S+\frac{h^{\alpha}}{\Gamma(1+\alpha)}\left[r S\left(1-\frac{S+I}{K}\right)-\beta S I+\omega I\right] \\
I & =I+\frac{h^{\alpha}}{\Gamma(1+\alpha)}[\beta S I-(\omega+\delta) I] .
\end{aligned}
$$

Eqs (11) give three fixed points as follows.

1. The origin point $E_{0}=(0,0)$ which is always exists.

2. The disease-free point $E_{1}=(K, 0)$ which is always exists.

3. The endemic point $E_{2}=\left(0,\left(\frac{K}{R_{0}},\left(1-\frac{1}{R_{0}}\right) \frac{r K}{r+\delta R_{0}}\right)\right)$ which is exists if $R_{0}>1$ where $R_{0}$ is the ratio reproduction number given by $R_{0}=\frac{\beta K}{\omega+\delta}$, see [6].

These fixed points are similar with its fractional-order model (1) in [6]. Now, we study the dynamics of these fixed points by considering the influence of the given step size $(h)$ occurs from the PWCA process.

Theorem 3. Let $h_{0}=\sqrt[\alpha]{\frac{2 \Gamma(1+\alpha)}{\omega+\delta}}$. The origin point $E_{0}=(0,0)$ is

(i) A saddle if $h<h_{0}$; or

(ii) A source if $h>h_{0}$; or

(iii) A non-hyperbolic if $h=h_{0}$.

Proof. By evaluating the Jacobian matrix of model $(10)$ at $E_{0}=(0,0)$, we acquire

$$
\left.J(S, I)\right|_{E_{0}}=\left[\begin{array}{cc}
1+\left(\frac{h}{h_{0}}\right)^{\alpha} \frac{2 r}{(\omega+\delta)} & \left(\frac{h}{h_{0}}\right)^{\alpha} \frac{2 \omega}{(\omega+\delta)} \\
0 & 1-2\left(\frac{h}{h_{0}}\right)^{\alpha}
\end{array}\right] .
$$

The Jacobian matrix (12) gives two eigenvalues as follows. 


$$
\begin{aligned}
& \lambda_{1}=1+\left(\frac{h}{h_{0}}\right)^{\alpha} \frac{2 r}{(\omega+\delta)}, \\
& \lambda_{2}=1-2\left(\frac{h}{h_{0}}\right)^{\alpha} .
\end{aligned}
$$

It is clear that $\left|\lambda_{1}\right|>1$ and hence $E_{0}$ is always unstable. To identify more detail of the dynamics, we study the sign of $\lambda_{2}$. When $h<h_{0}, h>h_{0}$, and $h=h_{0}$, we have $\left|\lambda_{2}\right|<1$, $\left|\lambda_{2}\right|>1$, and $\left|\lambda_{2}\right|=1$, respectively. In accordance with Lemma 1 , the dynamics of $E_{0}$ given by Theorem 3 are proven.

\section{Theorem 4. Suppose that}

$$
h_{a}=\sqrt[\alpha]{\frac{2 \Gamma(1+\alpha)}{r}} \text { and } h_{b}=\sqrt[\alpha]{\frac{2 \Gamma(1+\alpha)}{(\omega+\delta)\left(1-R_{0}\right)}}
$$

The disease-free point $E_{1}=(K, 0)$ is

(i) A sink if $h<h_{a}$ and $h<h_{b}$.

(ii) A source if $h>h_{a}$ and $h>h_{b}$.

(iii) A saddle if $h<h_{a}$ and $h>h_{b}$, or $h>h_{a}$ and $h<h_{b}$.

(iv) A non-hyperbolic if $h=h_{a}$ or $h=h_{b}$.

Proof. By evaluating the Jacobian matrix of model $(10)$ at $E_{1}=(K, 0)$, we acquire

$$
\left.J(S, I)\right|_{E_{1}}=\left[\begin{array}{cc}
1-2\left(\frac{h}{h_{a}}\right)^{\alpha} & \frac{2}{r}\left(\frac{h}{h_{a}}\right)^{\alpha}\left[\left(1-R_{0}\right) \omega-\left(r+\delta R_{0}\right)\right] \\
0 & 1-2\left(\frac{h}{h_{b}}\right)^{\alpha}
\end{array}\right] .
$$

The eigenvalues obtained from the Jacobian matrix (13) are

$$
\begin{aligned}
& \lambda_{1}=1-2\left(\frac{h}{h_{a}}\right)^{\alpha}, \\
& \lambda_{2}=1-2\left(\frac{h}{h_{b}}\right)^{\alpha} .
\end{aligned}
$$

If $h<h_{a}, h>h_{a}$, and $h=h_{a}$, then $\left|\lambda_{1}\right|<1,\left|\lambda_{1}\right|>1$, and $\left|\lambda_{1}\right|=1$, respectively. Furthermore If $h<h_{b}, h>h_{b}$, and $h=h_{b}$, the eigenvalues $\lambda_{2}$ will satisfy $\left|\lambda_{2}\right|<1$, $\left|\lambda_{2}\right|>1$, and $\left|\lambda_{2}\right|=1$ respectively. Thus, by applying Lemma 1, we have the complete dynamics given by Theorem 4 .

\section{Theorem 5. Suppose that}




$$
\begin{array}{ll}
h_{1}=\sqrt[\alpha]{\frac{4 \Gamma(1+\alpha) R_{0}}{r\left(\omega \xi R_{0}+1\right)+\sqrt{\Delta}},} \quad h_{3}=\sqrt[\alpha]{\frac{4 \Gamma(1+\alpha) R_{0}}{r\left(\omega \xi R_{0}+1\right)-\sqrt{\Delta}}}, \\
h_{2}=\sqrt[\alpha]{\frac{\left(\omega \xi R_{0}+1\right) \Gamma(1+\alpha) R_{0}}{\left(R_{0}-1\right) \beta K}}, & \Delta=r^{2}\left(\omega \xi R_{0}+1\right)^{2}-4\left(R_{0}-1\right) \beta r K .
\end{array}
$$

If $R_{0}>1$, them the endemic point $E_{2}=\left(0,\left(\frac{K}{R_{0}},\left(1-\frac{1}{R_{0}}\right) \frac{r K}{r+\delta R_{0}}\right)\right)$ is

(i) A sink if $\Delta \geq 0$ and $h \in\left(0, h_{1}\right)$, or $\Delta<0$ and $h \in\left(0, h_{2}\right)$.

(ii) A source if $\Delta \geq 0$ and $h>h_{3}$, or $\Delta<0$ and $h>h_{2}$.

(iii) A saddle if $\Delta \geq 0$ and $h \in\left(h_{1}, h_{3}\right)$.

(iv) A non-hyperbolic if $\Delta \geq 0$ and $h=h_{1}$ or $h=h_{3}$, or $\Delta<0$ and $h=h_{2}$.

Proof. We first compute the Jacobian matrix of model (10) at fixed point $E_{2}$ as follows.

$$
\left.J(S, I)\right|_{E_{2}}=\left[\begin{array}{cc}
1-\frac{h^{\alpha} r}{\Gamma(1+\alpha) R_{0}}\left[\omega \xi R_{0}+1\right] & -\frac{h^{\alpha}\left(R_{0}-1\right)}{\Gamma(1+\alpha) \xi R_{0}} \\
\frac{h^{\alpha} \beta \xi r K}{\Gamma(1+\alpha) R_{0}} & 1
\end{array}\right],
$$

where $\xi=\frac{R_{0}-1}{r+\delta R_{0}}$. In order to use Lemma 1 and 2, we compute trace and determinant of Jacobian matrix $\left.J(S, I)\right|_{E_{2}}$. We obtain

$$
\begin{aligned}
\operatorname{Tr}\left(\left.J(S, I)\right|_{E_{2}}\right) & =2-\frac{h^{\alpha} r}{\Gamma(1+\alpha) R_{0}}\left[\omega \xi R_{0}+1\right] \\
\operatorname{Det}\left(\left.J(S, I)\right|_{E_{2}}\right) & =1-\frac{h^{\alpha} r}{\Gamma(1+\alpha) R_{0}}\left[\omega \xi R_{0}+1\right]+\frac{h^{2 \alpha}\left(R_{0}-1\right) \beta r K}{\Gamma^{2}(1+\alpha) R_{0}^{2}} .
\end{aligned}
$$

It is clear that $F(1)=\frac{h^{2 \alpha}\left(R_{0}-1\right) \beta r K}{\Gamma^{2}(1+\alpha) R_{0}^{2}}>0$, which means the necessary condition of Lemma 2 is satisfied. We also have the characteristic equation of Jacobian matrix $\left.J(S, I)\right|_{E_{2}}$ as follows.

$$
\lambda^{2}-\operatorname{Tr}\left(\left.J(S, I)\right|_{E_{2}}\right) \lambda+\operatorname{Det}\left(\left.J(S, I)\right|_{E_{2}}\right)=0,
$$

which gives eigenvalues

$$
\lambda_{1,2}=1-\frac{\left(r\left(\omega \xi R_{0}+1\right) \pm \sqrt{\Delta}\right) h^{\alpha}}{2 \Gamma(1+\alpha) R_{0}} .
$$


By applying Lemma 1 and Lemma 2, the dynamical behaviors given in Theorem 5(i-iv) are completely proven.

\section{Bifurcations}

In this section, we study the existence of one parameter bifurcation of fixed point of model (10) including forward, period-doubling, and Neimark-Sacker bifurcations. These bifurcation phenomena are the change in stability around the fixed point driven by a parameter. Since model (10) is an epidemiological model which constructed by PWCA, whe chose the infection rate $(\beta)$ and the step-size $(h)$ as the bifurcation parameters.

First, we discuss the existence of forward bifurcation driven by the infection rate $\beta$. The forward bifurcation is the condition when a stable fixed point losses its stability and the other stable fixed point occurs simultaneously when a parameter is varied. Denote from the existence condition of the fixed points, $E_{1}$ is always exists, while $E_{2}$ is exists if $R_{0}>$ 1. It is also confirmed that the value $h_{b}$ depends on $\beta$ while $h_{a}$ does not. Therefore, according to Theorem 4 , the stability of $E_{1}$ is changed when $\beta$ is varied. By simple algebraic computation, we acquire $h<h_{b}$ when $0<\beta<\beta^{*}$ with $\beta^{*}=\frac{(\omega+\delta)-2 \Gamma(1+\alpha)}{h^{\alpha} K}$. Although the stability of $E_{1}$ change sign when $\beta$ passes through $\beta^{*}$, we must find the condition so that $E_{2}$ occurs simultaneously when $E_{1}$ losses its stability. By considering the existence condition of $E_{2}$ and the stability condition of $E_{1}$, we ensure the step size $(h)$ must satisfies $h<\sqrt[\alpha]{1-\frac{2 \Gamma(1+\alpha)}{\omega+\delta}}$. Therefore, the existence condition of forward bifurcation is given by the following Lemma.

Lemma 6. Let $\beta^{*}=\frac{(\omega+\delta)-2 \Gamma(1+\alpha)}{h^{\alpha} K}$ and $h_{a}^{*}=\sqrt[\alpha]{1-\frac{2 \Gamma(1+\alpha)}{\omega+\delta}}$. Suppose that $h<$ $\min \left\{h_{a}, h_{a}^{*}\right\}$. The disease-free point $E_{1}=(K, 0)$ losses its stability via forward bifurcation when $\beta$ passes through $\beta^{*}$. 


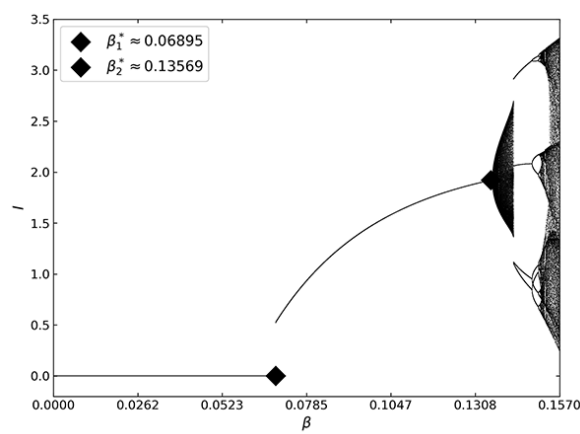

(a)

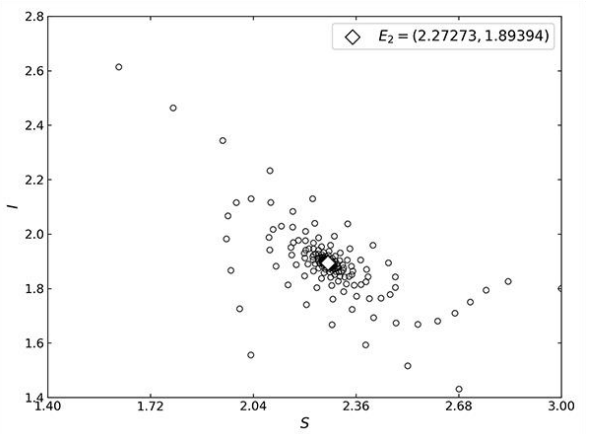

(c)

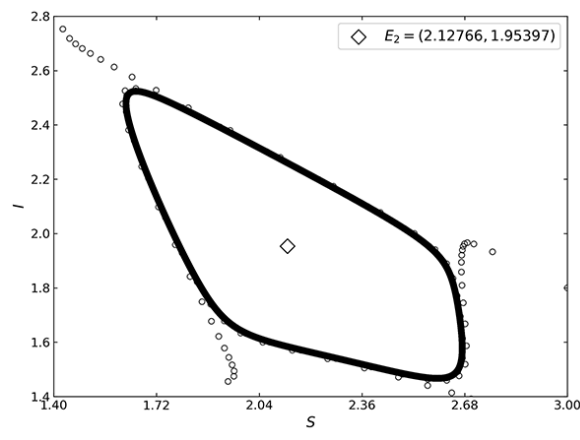

(e)

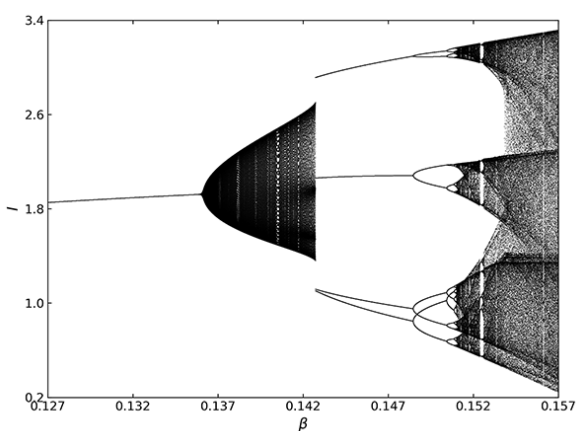

(b)

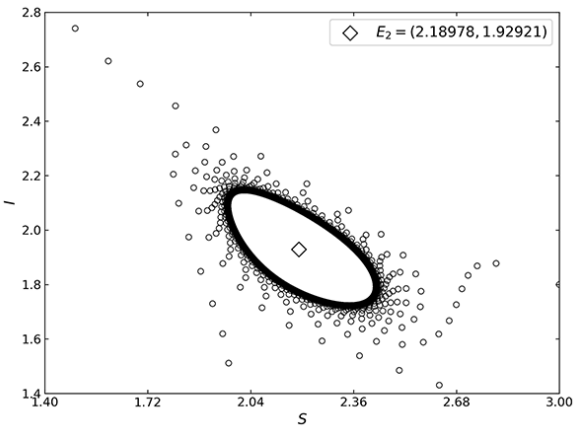

(d)

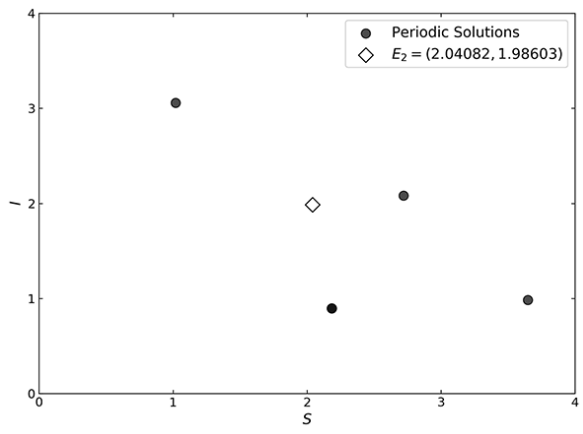

(f)

Figure 1 Numerical simulation of model (10) with parameter values (15), (a) bifurcation diagram in interval $0 \leq \beta \leq 0.157$, (b) local amplification to (a), (c,d,e) phase portrait when $\beta=0.132,0.137,0.141$ respectively, (f) period-4 solution when $\beta=0.147$

Now, we study the existence of period-doubling bifurcation both in $E_{1}$ and $E_{2}$ driven by the step-size $(h)$. The Period-doubling bifurcation is a circumstance where a stable (or sink) fixed point becomes unstable and converge to period-2 solution when a parameter is varied. This bifurcation exists when a single eigenvalue of the Jacobian matrix of a fixed point becomes equal to -1 [23],[24]. From Theorem $4, E_{1}$ is non-hyperbolic when 
$h=h_{a}$ or $h=h_{b}$. We affirm that $h_{a}>0$, and hence $h_{a}$ does not a bifurcation point. Furthermore, when $h=h_{b}$, an eigenvalue becomes equal to -1 . Therefore, the following Lemma is achieved.

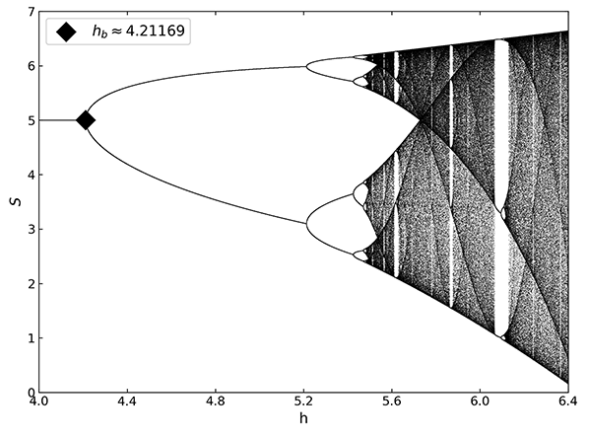

(a)

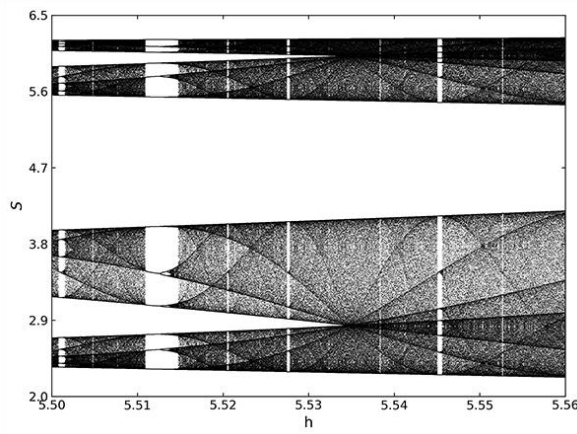

(c)

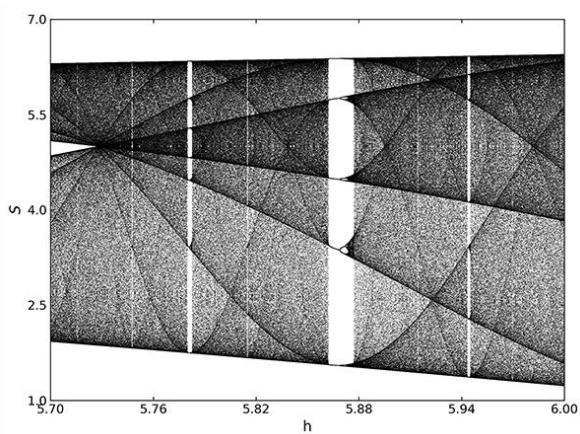

(e)

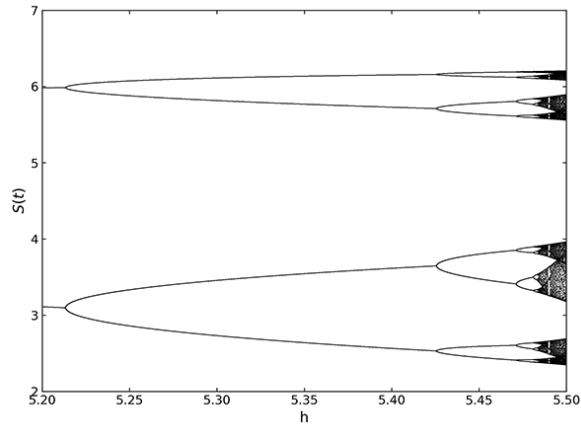

(b)

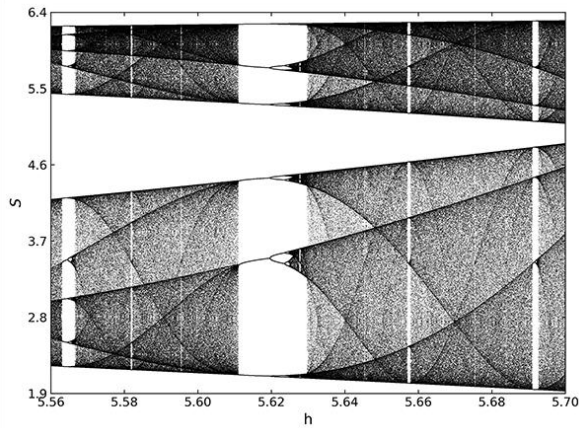

(d)

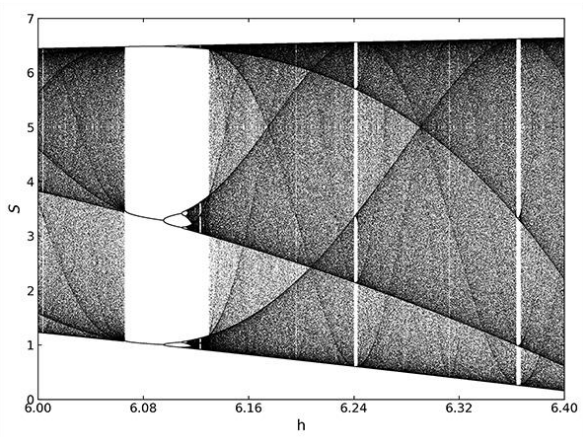

(f)

Figure 2 Numerical simulation of model (10) with parameter values (16), (a) bifurcation diagram in interval $4 \leq h \leq 6.4$, (b,c,d,e,f) local amplification to (a)

Lemma 7. The disease-free point $E_{1}=(K, 0)$ undergoes the period-doubling bifurcation when the step-size $(h)$ passes through $h_{b}$. 
By using the similar argument, we investigate the existence of bifurcation around the endemic point $E_{2}$, we confirm that the period doubling bifurcation may occurs around $E_{2}$ when $\Delta \geq 0$ and $h$ passes through $h_{1}$ or $h_{3}$.

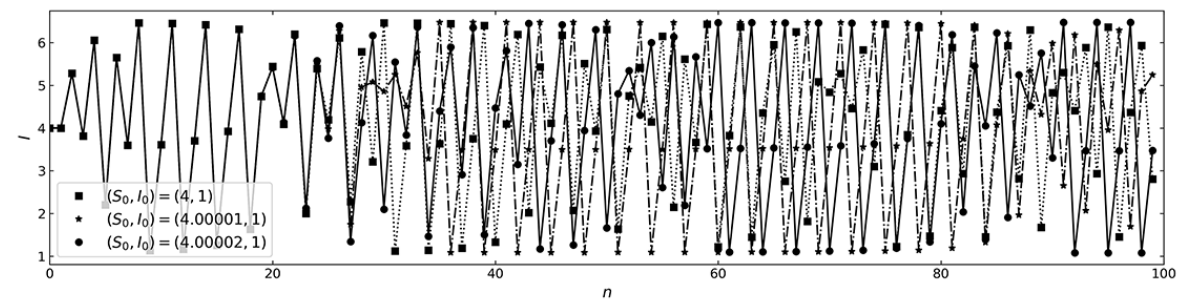

Figure 3 Time series of model (10) with parameter values (16) and $h=6.0656$. The chaotic solutions occur near period-3 solution.

Next, we discuss the occurrence of Neimark-Sacker bifurcation around the endemic point $E_{2}$. Neimark-Sacker bifurcation is a condition when a stable (sink) fixed point losses its stability and a stable limit-cycle occurs simultaneously if a parameter is varied. This bifurcation is pretty similar with Hopf bifurcation in the continuous model. Now, we will show the existence of Neimark-Sacker bifurcation driven by the step-size $(h)$. This bifurcation occurs when we have a pair of complex conjugate eigenvalues with modulus 1 , see [23] and [24]. It is confirmed that when $\Delta<0$ and $h=h_{2}$, we have a pair of complex conjugate eigenvalues with modulus 1 . Therefore, the existence of both bifurcations around $E_{2}$ are given by the following Lemma.

Lemma 8. The endemic point $E_{2}=\left(0,\left(\frac{K}{R_{0}},\left(1-\frac{1}{R_{0}}\right) \frac{r K}{r+\delta R_{0}}\right)\right)$ undergoes

(i) a period-doubling bifurcation when $\Delta \geq 0$ and $h$ passes through $h_{1}$ or $h_{3}$.

(ii) a Neimark-Sacker bifurcation when $\Delta<0$ and $h$ passes through $h_{2}$.

\section{$5 \quad$ Numerical Simulations}

In this section, we explore the dynamics of model (10) numerically, including the existence of forward, period-doubling, and Neimark-Sacker bifurcations. We divide the simulation into two parts i.e., the influence of the Infection Rate $(\beta)$ and the step-size (h). These two parameters are chosen according to the theoretical results, where the dynamics of model (10) are influenced by the parameter values of $\beta$ and $h$.

\subsection{The influence of the Infection Rate $(\beta)$}

The influence of the infection rate $(\beta)$ is investigated numerically by first set the parameter values as follows.

$$
r=0.5, \quad K=5, \quad \omega=0.2, \quad \delta=0.1, \quad h=5 .
$$


In fig. 1a, we portray the bifurcation diagram and some phase portraits of model (10) in interval $0 \leq \beta \leq 0.157$. When $0<\beta<\beta_{1}^{*}$, where $\beta_{1}^{*} \cong 0.06895$, the disease-free point $E_{1}$ is a sink and losses its stability after surpasses $\beta_{1}^{*}$. A sink $E_{2}$ also occurs simultaneously after $\beta$ crosses $\beta_{1}^{*}$, this stability of $E_{2}$ is maintained in interval $\beta_{1}^{*}<\beta<\beta_{2}^{*}$, where $\beta_{2}^{*} \cong$ 0.13569 . This circumstance confirms the stability condition in Theorem 4(i) and the existence of forward bifurcation in Lemma 6.

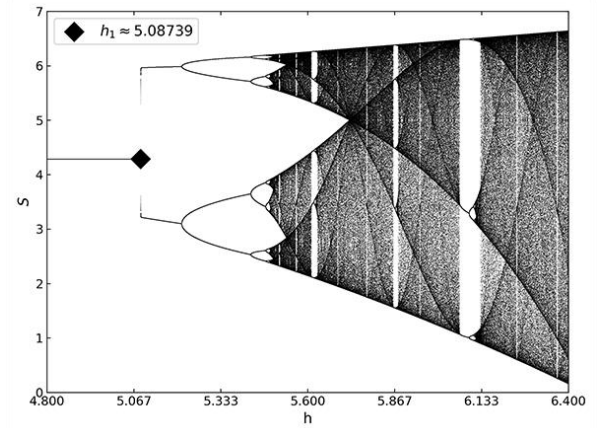

(a)

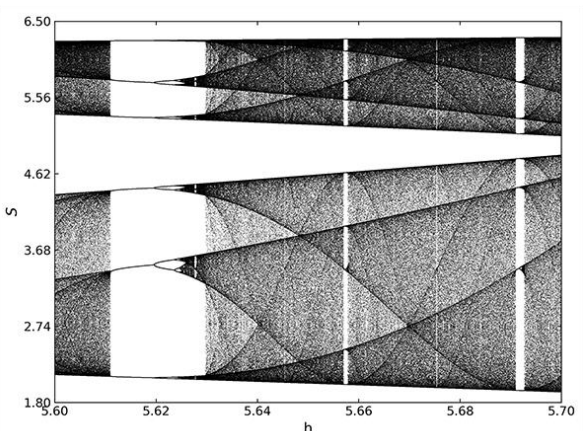

(c)

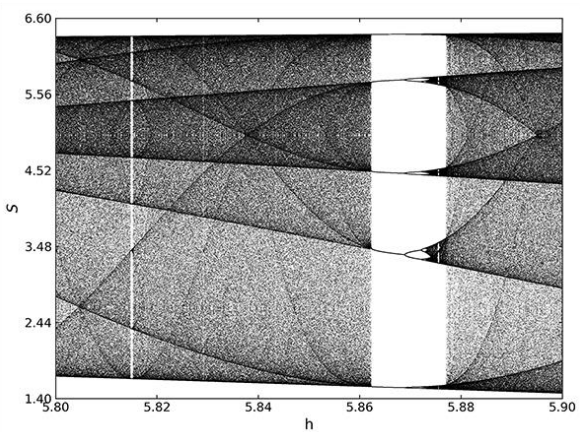

(e)

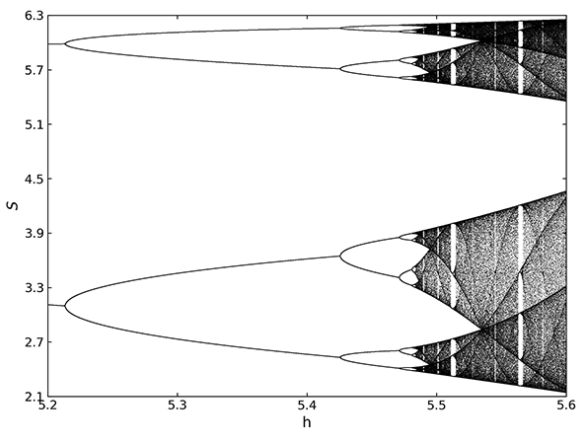

(b)

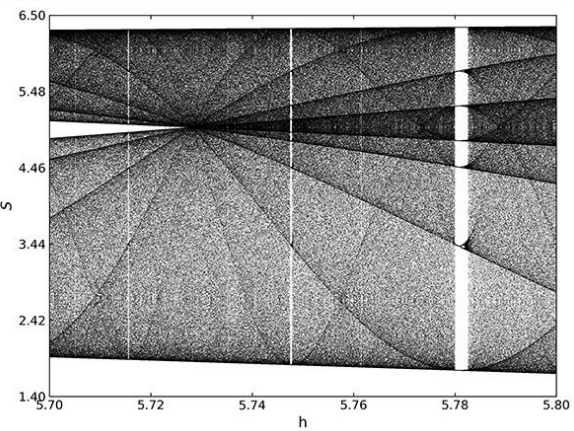

(d)

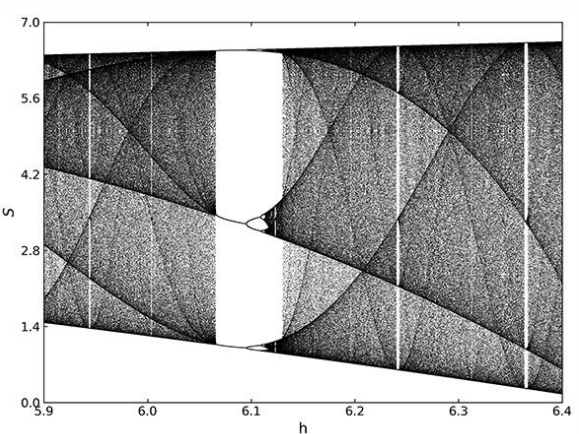

(f)

Figure 4 Numerical simulation of model (10) with parameter values (17), (a) bifurcation diagram in interval $4.8 \leq h \leq 6.4$, (b,c,d,e,f) local amplification to (a) 


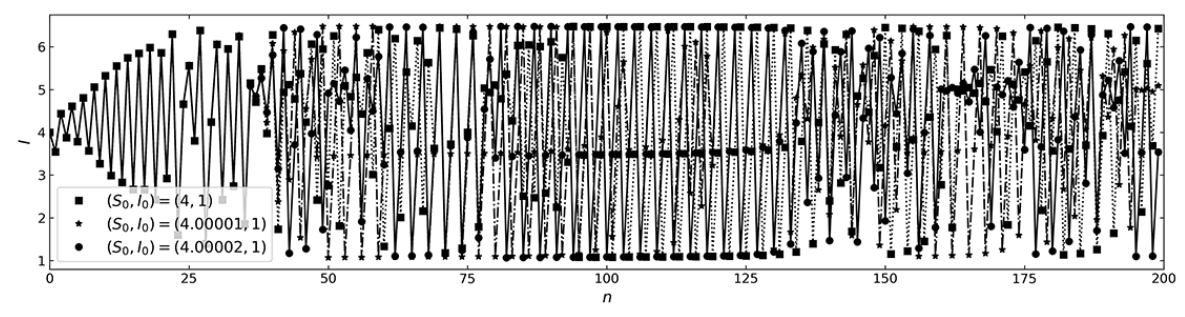

Figure 5 Time series of model (10) with parameter values (17) and $h=6.065$. The chaotic solutions occur near period-3 solution.

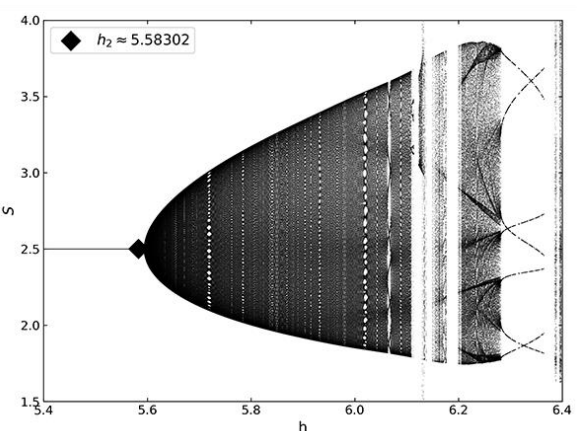

(a)

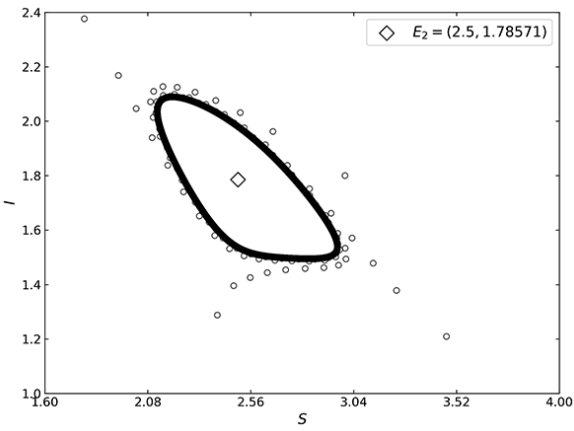

(c)

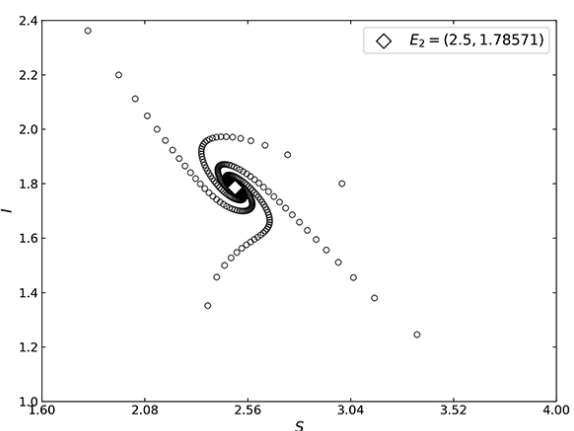

(b)

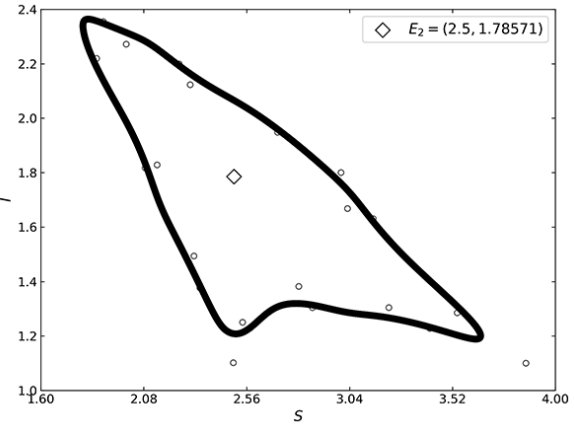

(d)

Figure 6 Numerical simulation of model (10) with parameter values (18), (a) bifurcation diagram in interval $5.4 \leq h \leq 6.4,(\mathbf{b}, \mathbf{c}, \mathbf{d})$ phase portraits around $E_{2}$ with $h=5.55,5.7$, 6.1 , respectively

The other bifurcation also occurs when $\beta$ passes through $\beta_{2}^{*}$, see fig. $1 \mathrm{~b}$. The endemic point $E_{2}$ undergoes a Neimark-Sacker bifurcation driven by parameter $\beta$. When $\beta_{1}^{*}<$ $\beta<\beta_{2}^{*}, E_{2}$ is a sink, and when $\beta>\beta_{2}^{*}, E_{2}$ becomes a source and a stable limit-cycle occurs. To confirm the dynamics, we set $\beta=0.132$ and $\beta=0.137$ and give the phase 
portraits as in fig. $1 \mathrm{c}$ and $1 \mathrm{~d}$. Furthermore, we increase the infection rate to $\beta=0.141$. We find that the diameter of the limit-cycle is getting bigger, see fig.1e. Now, we increase more the value of the infection rate to $\beta=0.147$ and the limit-cycle vanish and replaced by a stable period-4 solution.

\subsection{The influence of the Step-Size $(h)$}

In this part, the influence of $h$ to the dynamics of model (10) are discussed both for $E_{1}$ and $E_{2}$. We start investigate the dynamics of $E_{1}$ by setting the parameter as follows.

$$
r=0.5, \quad K=5, \quad \beta=0.05, \quad \omega=0.2, \quad \delta=0.1 \text {. }
$$

We use parameter (16) to portray the bifurcation diagram driven by the step-size $(h)$ in interval $4 \leq h \leq 6.4$ and achieve fig. 2 a. The fixed point $E_{1}$ undergoes a period-doubling bifurcation when $h$ crosses $h_{b} \cong 4.21169$, which confirm the analytical results given by Lemma 7. We also show the existence of some stable periodic solutions which also occurs via period-doubling bifurcation. In fig. $2 b$, for interval $5.2 \leq h \leq 5.5$ each branch of period-2 solution bifurcated to period-4,8,16 and so forth. Next, in interval $5.5 \leq h \leq 5.6$ (see fig. 2c), we find the periodic solution with period-12,14,16 and period-20. For interval $5.6 \leq h \leq 5.7$ (fig. 2d), we have period-6,8,10 solution and for interval $5.7 \leq$ $h \leq 6$ (fig.2e) some solutions converge to period-5 and 7. The interesting phenomenon occurs in interval $6 \leq h \leq 6.4$, see fig. $2 \mathrm{e}$. There exists a period-3 solution which leads to chaotic solution [23]. We set $h=6.0656$ where according to fig.2e, this value of stepsize is very close to period-3 solution. We chose three very close initial values i.e., $\left(S_{0}, I_{0}\right)=(4,1),\left(S_{0}, I_{0}\right)=(4.00001,1)$, and $\left(S_{0}, I_{0}\right)=(4.00002,1)$ and plot the timeseries in fig. 3. Denote that the solutions are also very close for $n \in[0,25)$ and becomes separated when the time increase. This condition indicated the existence of chaotic solution.

Now, we study the dynamics around $E_{2}$. we set the parameter values as follows.

$$
r=0.5, \quad K=5, \quad \beta=0.07, \quad \omega=0.2, \quad \delta=0.1 .
$$

The bifurcation diagram using parameter values (17) is given by fig. 4a. The stable endemic point $E_{2}$ becomes unstable via period-doubling bifurcation. This bifurcation point is $h_{1}=5.09739$ which associated with Lemma 8(i). As the dynamics of $E_{1}$ when period-doubling occurs, there are some periodic solutions also appears around $E_{2}$. From fig $4 \mathrm{~b}$ to $4 \mathrm{f}$, we have period- 2 until period-10 solutions and so forth. The chaotic solution also exists denoted by the occurrence of period-3 solution. When $h=6.065$, we plot some solutions with adjacent initial values, see fig. 5. For $n \in[0,40)$, the distance between solution still maintained, but the solution becomes chaos when $n \rightarrow \infty$.

Finally, we set the parameter values as follows. 


$$
r=0.5, \quad K=5, \quad \beta=0.07, \quad \omega=0.2, \quad \delta=0.1 .
$$

For $h \in[5.4,6.4]$, we portray the bifurcation diagram in fig. 6a. We obtain numerically that $E_{2}$ undergoes a Neimark-Sacker bifurcation when $h$ passes through $h_{2}$ where $h_{2} \cong$ 5.58302. This numerical simulation strengthens the analytical results given by Lemma 8(ii). We also give the phase portraits to show the dynamics for some values of $h$. When $h=5.55, E_{2}$ is a sink (fig. 6b), when $h=5.7, E_{2}$ is a source and stable limit-cycle occurs, and when $h=6.1, E_{2}$ still a source but the diameter of limit-cycle increases.

\section{Conclusion}

The dynamics of a discrete fractional-order logistic growth model with infectious disease has been studied. The discrete model is obtained by piecewise constant argument (PWCA). By considering the previous work in [6], we ensure that its discrete model (10) has rich dynamics rather than the fractional-order derivative model (1). From [6], when $R_{0}<1$, the disease disappeared, while in the discrete model (10), there is condition makes the disease still exists and change periodically. When $R_{0}>1$, model (1) show that the disease exists and converges to the endemic point. The discrete model (10) show that we have other two optional conditions i.e., the endemic point becomes unstable via period-doubling or Neimark-Sacker bifurcation. When period-doubling bifurcation occurs, both susceptible and infected populations change periodically, while when Neimark-Sacker bifurcation occurs, the density of both populations are bounded by a limit-cycle. These conditions are not available in the fractional-order derivative model (1). From the epidemiological point of view, we have another alternative in presenting the behavior of infectious the disease.

\section{$7 \quad$ References}

[1] Zhang, F.W. \& Nie, L.F., 2017, Dynamics of SIS epidemic model with varying total population and multivaccination control strategies, Stud. Appl. Math., 139(4) 533550.

[2] Kermack, W.O. \& McKendrick, A.G., 1927, A contribution to the mathematical theory of epidemics, Proc. R. Soc. London. Ser. A, Contain. Pap. a Math. Phys., 115(772), 700-721.

[3] Dos Santos, J.P.C., Monteiro, E., \& Vieira, G.B., 2017, Global stability of fractional SIR epidemic model, 5, 1-7.

[4] Hassouna, M., Ouhadan, A., \& El Kinani, E.H., 2018, On the solution of fractional order SIS epidemic model, Chaos Soliton Fract., 117, 168-174.

[5] Hoang, M.T., Zafar, Z.U.A., \& Ngo, T.K.Q., 2020, Dynamics and numerical approximations for a fractional-order SIS epidemic model with saturating contact rate, Comput. Appl. Math., 39(4), 277.

[6] Panigoro, H.S. \& Rahmi, E., 2020, Global stability of a fractional-order logistic growth model with infectious disease, Jambura J. Biomath., 1(2), 49-56. 
[7] Widya, E., Miswanto, M., and Alfiniyah, C., 2020, Analisis kestabilan model matematika penyebaran penyakit schistosomiasis dengan saturated incidence rate, Contemp. Math. Appl., 2(2), 71-88.

[8] Ahaya, S. O. S. P., Rahmi, E., and Nurwan, N., 2020, Analisis dinamik model SVEIR pada penyebaran penyakit campak, Jambura J. Biomath., 1(2), 57-64.

[9] Jajarmi, A., Yusuf, A., Baleanu, D., and Inc, M. , 2019, A new fractional HRSV model and its optimal control: A non-singular operator approach, Phys. A Stat. Mech. its Appl., 547, 123860.

[10] Fatmawati, Khan, M. A., Alfiniyah, C., and Alzahrani, E., 2020, Analysis of dengue model with fractal-fractional Caputo-Fabrizio operator, Adv. Differ. Equations, 2020(1), 422.

[11] Okyere, E., Ackora-Prah, J., and Oduro, F. T. , 2020, A Caputo based SIRS and SIS fractional order models with standard incidence rate and varying population, Commun. Math. Biol. Neurosci., 2020(60).

[12] Abdelaziz, M. A. M., Ismail, A. I., Abdullah, F. A., and Mohd, M. H. , 2018, Bifurcations and chaos in a discrete SI epidemic model with fractional order, Adv. Differ. Equations, 2018(1), 44.

[13] Shi, Y., Ma, Q., and Ding, X., 2018, Dynamical behaviors in a discrete fractionalorder predator-prey system, Filomat, 32(17), 5857-5874.

[14] Elettreby, M. F., Ahmed, E., and Alqahtani, A. S., 2020, A discrete fractional-order Prion model motivated by Parkinson's disease," Math. Probl. Eng., 2020, 1-12.

[15] Agarwal, R. P., El-Sayed, A. M. A., and Salman, S. M. , 2013, Fractional-order Chua's system: discretization, bifurcation and chaos, Adv. Differ. Equations, 2013(1), 320.

[16] El-Sayed, A. M. A. and Salman, S. M., 2013, On a discretization process of fractional order Riccati differential equation, J. Fract. Calc. Appl., 4(2), 251-259.

[17] Podlubny, I., 1999, Fractional differential equations: an introduction to fractional derivatives, fractional differential equations, to methods of their solution and some of their applications, San Diego CA: Academic Press.

[18] Diethelm, K., 2010, The analysis of fractional differential equations: an application-oriented exposition using differential operators of caputo type, Berlin, Heidelberg: Springer.

[19] El Raheem, Z. F. \& Salman, S. M., 2014, On a discretization process of fractionalorder Logistic differential equation, J. Egypt. Math. Soc., 22(3), 407-412.

[20] Elsadany, A. A. \& Matouk, A. E., 2015, Dynamical behaviors of fractional-order Lotka-Volterra predator-prey model and its discretization, J. Appl. Math. Comput., 49(1-2), 269-283.

[21] Din, Q., Elsadany, A. A., \& Khalil, H., 2017, Neimark-sacker bifurcation and chaos control in a fractional-order plant-herbivore model, Discret. Dyn. Nat. Soc., 2017(3), Article ID 6312964. 
[22] Mokodompit, R., Nurwan, and Rahmi, E., 2020, Bifurkasi periode ganda dan Neimark-Sacker pada model diskret Leslie-Gower dengan fungsi respon ratiodependent," Limits J. Math. Its Appl., 17(1), 19.

[23] Elaydi S., Discrete Chaos with Applications in Science and Engineering, 2nd ed, Boca Raton: Chapman and Hall/CRC; 2008.

[24] Singh, A., Elsadany, A. A., and Elsonbaty, A., 2019, Complex dynamics of a discrete fractional-order Leslie-Gower predator-prey model, Math. Methods Appl. Sci., 42(11), 3992-4007. 